\title{
MICROBIOME OF AQUATIC VERTEBRATES WITH SPECIAL REFERENCE TO FISH
}

\author{
G. DASH ${ }^{1 *}$, K. B. SANYAL ${ }^{1}$, D. MUKHERJEE ${ }^{1}$ AND P. MALI $^{1}$ \\ ${ }^{1}$ Department of Aquatic Animal Health, West Bengal University of Animal and Fishery Sciences, \\ Kolkata-700 094, West Bengal, India
}

\begin{abstract}
Studies on gut microbiome in aquatic animals is a field that has grown equivalently with the increase in aquaculture industry. Research in this field is progressing quickly focussing on nutritional manipulation, developments in microbiome research and modification of the gut microbiota. The outcome of the research is helpful in fish farming as well as to maintain host health and welfare. However, clear understanding of the specific role played by the different gut microbiota on diversified fish species is still lacking. A number of important topics have been highlighted here which would be beneficial for further research at potent areas.
\end{abstract}

Key words: Abiotic and biotic factors, Fish, Gut microbiota, Metabolism

\section{Introduction}

A microbiome includes all the microorganisms in a particular ecosystem. Occasionally it comprises the total of the genetic material of the microbes, symbiotic and pathogenic, living in and on all vertebrates. The exothermic aquatic vertebrates (i.e., fishes), and their associated microbiota are mostly unexplored. Minimum information exists on the biodiversity, geographic distribution, and seasonality of microbiota on marine, estuarine, and freshwater fish welfare (Sandrini et al., 2015).

Studies on fish gut microbiome may help to improve welfare of fish and aquaculture practices. On the other hand, remarkable differences between mammals and fish metabolism and energy expenditure (Sandrini et al., 2015) survive and host-microbe interactions and homeostasis disparity could be expected among fish and mammals.

The work relating to the gut microbiota of fish is dominated recently on humans and mammals.
This field has historical interest and grown significantly with the expansion of the aquaculture industry and developments in the research of microbiome. Major attention has been focusses towards nutritional manipulation and modification of the gut microbiota aiming to support the fish farming, as well as to maintain host health and welfare.Yet, there is a gap about the basic understanding of the role of gut microbiota (Egerton et al., 2018).

This review explains the role of the fish gut microbiota in regards to the host physiology, feeding, digestion and metabolism, and also its control over stress responses, reproduction, development, and immune responses. Environmental and host-specific factors are also discussed, in addition to future implications of fish gut microbiota handling and possible research guidelines for this growing field.

\section{Gut microbiota}

Gut or gastrointestinal (GI) tract is an organ that starts from the mouth to the anus. It helps in 
digestion and absorption of nutrients and expels the remaining waste as feces (Tarnecki et al., 2017). Autochthonous (adherent) bacteria in the fish gut take part in digestion processes. For the examination of gut microbiota of fish, feces used to analyze allochthonous (non-adherent) bacteria and intestinal rinsed was used to identify autochthonous bacteria (there were significant variances in the microbiota of feces). Generally, loads of bacteria in intestine remained ranging from $10^{2}$ to $10^{9} \mathrm{CFU} / \mathrm{g}$ (Tarnecki et al., 2017). Allochthonous bacteria typically are more numerous than autochthonous bacteria. It was observed that feces have varieties of microbial flora than rinsed intestine samples. Gut microbiota in marine fish predominantly include Vibrio, Pseudomonas, Acinetobacter,Corynebacterium, Alteromonas, Flavobacterium and Micrococcus (Zhou et al., 2009). In freshwater fish, the gut microbiota observed are Aeromonas, Pseudomonas, Enterobacteriaceae, Micro-coccus, Acinetobacter, Clostridium, Bacteroides type $\mathrm{B}$ and Fusarium. In comparison with freshwater fish, marine fish maintains a superior number of Pseudoalteromonas and Vibrio in both feces and intestine and a higher incidence of Photobacterium in feces samples (Liu et al., 2016).

\section{Importance of fish microbiota}

During initial metamorphic stage, bacteria can play a vital role to host development and health. The gut microbiota is necessary for proper growth and functioning of immune system. For example, upregulation of genes associated in innate immunity occurs in the presence of commensal microbes. Gut microbiota influences adequate growth and maturation of the gut-associated lymphoid tissues which control immune functions in the GI tract and additionally provide protection against pathogens. The gut microbiota plays a critical role in host nutrition from metabolizing compounds and producing vitamins to aid the host in the uptake of nutrients. Gut microbes help to metabolise the cellulose and other complex polysaccharides as fish have the lack of endogenous cellulase enzyme. Gut microbiota can generate vitamin B12 (Cyanocobalamin) in freshwater. Some gut bacteria can produce short-chain fatty acids as metabolic end product that the host can use for energy or lipid synthesis (Falcinelli et al., 2018).

\section{Physiological roles of gut microbiota}

Feeding/digestion/metabolism: A few studies have been conducted in fish microbiota influencing feeding and metabolism, but there are evidences of similarities with mammals (Fetissov, 2017). In some studies the influence of microbiota on food intake has been observed to compare feeding rates and changes in microbiota composition, though results are incompatible and difficult to compare. Zebrafish fed with Lactobacillus rhamnosus have reduced appetite compared to control fish (Falcinelli et al., 2018). However, carp fed with fructooligosaccharide (FOS) diet supplement exhibit changes in microbiota composition compared to fish fed with the control diet (Hoseinifar et al., 2014).

The effect of gut microbiome on metabolism in few fish species has been examined. Several biosynthesis, and metabolism pathways of carbohydrates, amino acids and lipids are influenced in grass carp (Ctenopharyngodon idella), because the colonization of gut microbiota (Semova et al., 2012). In zebrafish, the colonization of the gut microorganisms promotes epithelial absorption of fatty acids (Sheng et al., 2018). Fish with intact microbiota have increased lipid accumulation in the intestinal epithelium compared to fish who lacks microbiota (Sandrini et al., 2015). Compared to fish fed with control diets, Japanese flounder (Paralichthys olivaceus) fed a diet supplemented with Bacillus clausii exhibited higher weight gain, feed effectiveness and growth performance. All of these data suggest that gut microbiota has strong control in metabolism of fish.

The gut microbiota discharges metabolites in reaction to substrates present in the gut lumen. 
These metabolites stimulate the enteroendocrine cells of the GI tract and reach the brain (Fig. 1). The stimulated enteroendocrine cells discharge gut peptides, act in the GI tract and affect brain feeding centers, altering neuropeptide release, and modifying feeding behavior and energy homeostasis (Butt and Volkoff, 2019).

Stress response: The stress response is mediated by a number of hormones and is a result of the bi-directional communication between the brain and peripheral organs (Sandrini et al., 2015). Stress in fish can be caused by a number of environmental factors like poor water quality, high levels of particulates, oxygen levels, temperature, high population density, malnutrition (Cantas et al., 2012). When stress occurs, the hypothalamic-pituitary-adrenal (HPA) axis releases corticotrophin-releasing hormone $(\mathrm{CRH})$ that stimulates the anterior pituitary for secretion of adrenocorticotropic hormone $(\mathrm{ACTH})$ which ultimately stimulates the secretion of adrenal glucocorticoids to prepare the body to cope with stress. In fish, as in mammals (Sudo, 2014), the microbiome affects the HPA axis, the stress response and behavior, in particular, anxiety-like and locomotor behaviors, which might in turn affect feeding behavior and energy homeostasis. For example, in zebrafish, the microbiota reduces anxiety-like behavior (Davis et al., 2016) and decreases the stress response by lowering CRH expression and cortisol levels. Disruption of the gut microbiota might thus decrease the ability of the fish to forage for food and decrease feeding by increasing levels of stress hormones, which have been shown to inhibit feeding [e.g., rainbow trout (Oncorhynchus mykiss); goldfish (Carassius auratus)].

In fish, acute stress such as netting stimulates an increased sloughing off of mucus and it causes removal of autochthonous bacteria which play a protective role against pathogens (Vatsos, 2016). Largely, stress results in alterations of gut microbiota resulting to decrease in feed intake as observed in Chinook salmon (Oncorhynchus tshawytscha) (Bernier, 2010). However, different fish species may survive with stress in different ways, so that the effects of stress on gut microbiota may vary among species.

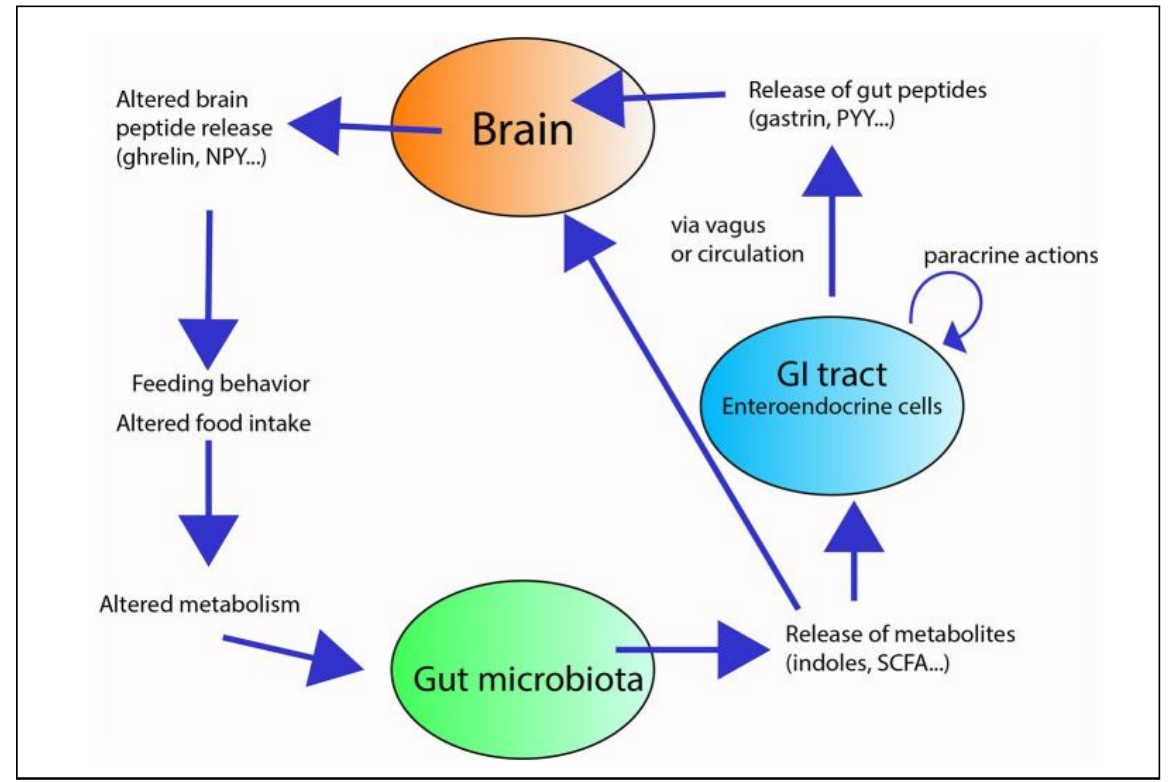

Fig. 1. Overview of the gut-microbiota-brain axis in feeding and digestion (Source: Butt and Volkoff, 2019) 
Reproduction: Reproduction is directly related to energy homeostasis, and can only be successfully completed when sufficient energy stores are available (Carnevali et al., 2017). It has been observed that probiotic administration on fish gonad may enhance the reproductive performance of the host. For example, when Lactobacillus rhamnosus had been administered continuously from birth to sexual maturation, it altered the gut microbiota and accelerated larval development of zebrafish by improving growth and sex differentiation (Avella et al., 2012). Adult female zebrafish treated with L. rhamnosus display an increase in the number of vitellogenic follicles and higher gonadosomatic indexes (GSI). Similar findings were also observed in ornamental fishes species viz. livebearer fish species and goldfish (Mehdinejad et al., 2018) when their feed supplemented with probiotic increases GSI, fecundity and fry production of spawning females and length and weight of fry though the mechanisms mediating the actions of gut microbiota in host reproduction are still under study.

Development: The development of tissues and organs structure in an animal is largely influenced by the presence and composition of the microbiota and it is particularly observed in the development of digestive system (Stephens et al., 2016).

The composition of gut microbiota showed a clear pattern of host development (Yan et al., 2016). In grass carp (Ctenopharyngodon idella), bacterial communities differ in eggs and the larvae and variety of bacteria population increases as the fish grows from egg to larvae (Wang et al., 2015). Intestinal cell proliferation during the period of development from the larval to the adult stage in different species of fishes like grass carp, Chinese perch (Siniperca chuatsi) and southern catfish (Silurus meridionalis), it has been demonstrated that the gut microbiota variation levels increase with the development of fish. Zebrafish model was used to study for effect of gut microbiota in epithelial cell proliferation and the workers suggested a direct evidence of the gut microbiota role in gartrointestinal tract (GIT) development (Cheesman et al., 2011).

Evidence suggests that the microbiota is also involved in the neurological development (e.g. zebrafish). Fish with microbiota disorder following antibiotic administration indicate an abnormal locomotive activity (Phelps et al., 2017), which might have an effect on feeding and foraging. The mechanisms behind this interaction are still unidentified.

Immune responses: Intestinal microbiota contribute to many aspects of host defence against invading pathogens both through direct microbial antagonism and promotion of maturation of the intestinal immune system. Bacterial metabolism results in the production of several byproducts with an antimicrobial effect, such as peroxides and various acids. Some of these substances do not just inhibit the growth of pathogenic microorganisms themselves, but might also potentiate the effectiveness of other antimicrobial substances (Xiong et al., 2019). Several types of sentinel cells, like M cells, luminal macrophages or dendritic cells (DCs) are sensing the pathogenic bacteria in the mucosal lumen and they play a key role in immune response to limit the colonization of pathogens in host (Ribet and Cossart, 2015). It has been suggested that the microbiota keep the host from colonization and proliferation of environ-mental pathogens, called "colonization resistance" (Lawley and Walker, 2013; Kim et al., 2017). Although the mechanism behind this resistance is yet to be explored. The associated commensal microbiota makes an important contribution to the host fish immunity and metabolism and the gut microbiota plays a major role in the development and maturation 
of the gut associated lymphoid tissue (GALT) (Lazado and Caipang, 2014; Wang et al., 2018). Administration of probiotics in rainbow trout and gilthead seabream (Sparus aurata) by Tapia-Paniagua et al. (2011) had improved both the intestinal microbiota and the immune response.

\section{Factors affecting fish gut microbiota}

Biotic (genotype, physiological status, pathobiology, life style) and abiotic (environmental) factors may affect the fish gut microbiota and control its composition and variety, with its function and metabolic activity, growth, energy storage and health of the fish (Ghanbari et al., 2015) (Fig. 2). This part will evaluate the intrinsic and extrinsic factors and give specific examples in which the gut microbiota of various fish has been altered as a result.

Environmental factors: Initially, fish embryos develop in a relative constant bacteria-free environment (within the egg or the mother). Fish

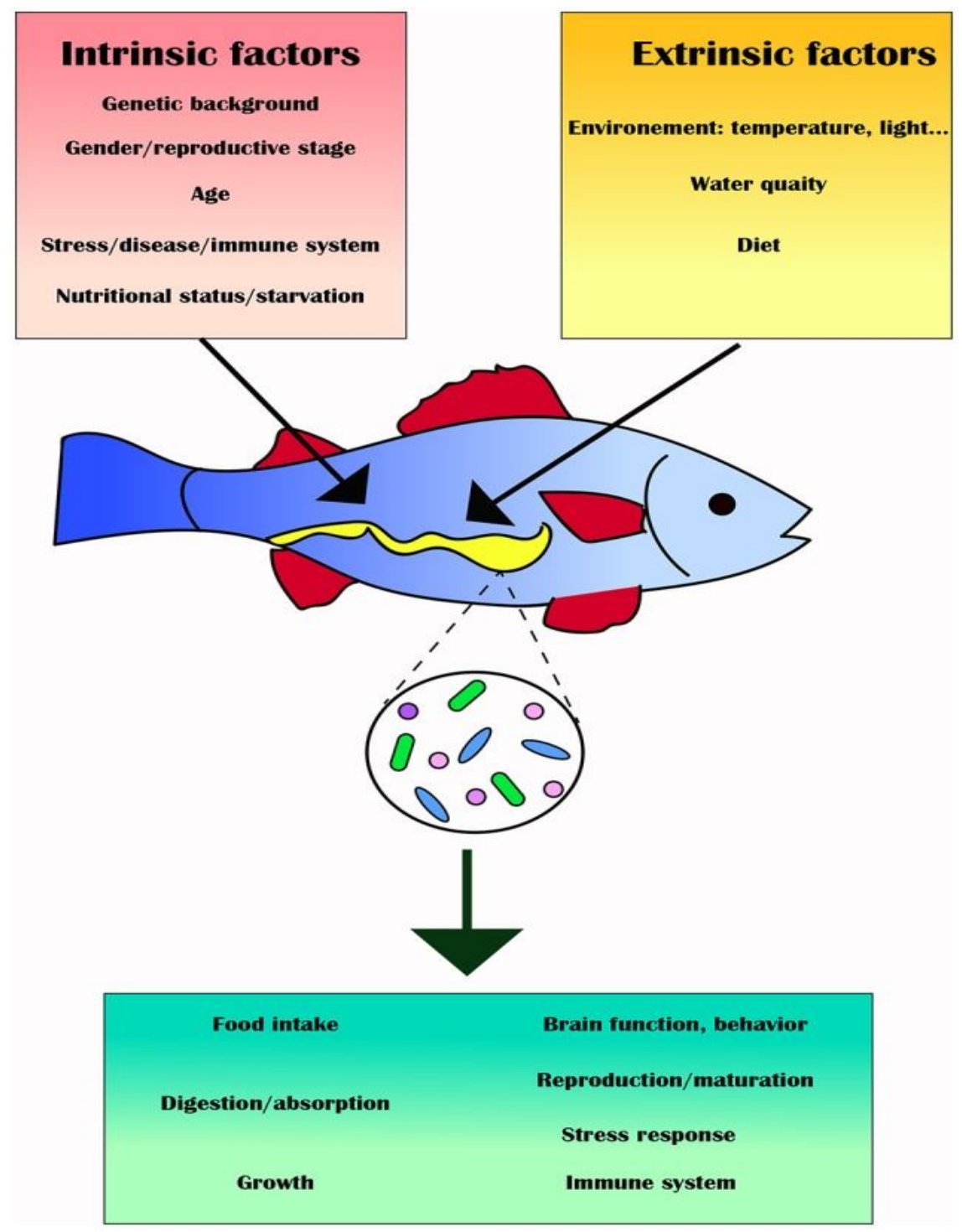

Fig. 2. Intrinsic and extrinsic factors can alter the gut microbiota and its downstream effects on the fish host (Source: Butt and Volkoff, 2019) 
are thus theoretically microbe-free at hatching and they obtain gut microbes from surrounding environments (Li et al., 2017). After hatching, environmental factors like water composition, quality and temperature significantly influence the gut microbiota throughout the life (Vatsos, 2016). It may be stated that compositions of bacteria fluctuate between fish inhabiting freshwater and marine ecosystems. Generally Aeromonas and Pseudomonas prevail in the freshwater fish, while in marine fish, Vibrio is the most common genus (Vatsos, 2016).

Different seasons stimulate food utilization due to variations in nutrient loads of water. As a result, the composition of the microbiota may vary with particular seasons. It is observed that gut bacterial load of tilapia decreases in winter compared to other seasons, and Pseudomonas, Micrococcus, and Flavobacterium are only present in the gut of hybrid tilapia (Oreochromis niloticus $\times$ Oreochromis aureus) in the winter (Al-Harbi and Uddin, 2004).

Rearing conditions have been shown to control the composition of the gut microbiota. Atlantic salmon held in two different holding conditions (indoor recirculating aquarium and cage culture in an open environment) have different compositions of gut microbiota (Dehler et al., 2017).

Environmental pollutants and toxins may also influence the fish microbiota. For example, common carp (Cyprinus carpio) exposed to waterborne copper (Meng et al., 2018) and zebrafish exposed to polystyrene microparticles show disturbances of the intestinal microbiota associated to immunity, these chemicals increase susceptibility to pathogens and inflammation. Other kinds of environmental chemicals; pesticides (Kan et al., 2015), heavy metals and antibiotics (Zhou et al., 2018) can affect gut microbiota thereby reducing the ability of absorbing nutrients.
Host-specific intrinsic factors: Fish microbiota composition varies due to several factors, including phylogeny, genetics and sex, age, and diet.

Genetics/sex- Genetic condition influences the gut microbiota (intra and inter-specific variations). Interspecies differences in the gut microbiota composition among individuals of the same species may be present. It has been observed that in rainbow trout, some bacterial groups are associated with specific families, which indicate the composition of gut microbiota was dependant on diet (Navarrete et al., 2012)

Fish sex may influence the gut microbiota through sex-specific host-microbe interactions, diet preferences or immune responses (Bolnick et al., 2014). For example, differences in the gut microbiota between sexes of the three spine stickleback (Gasterosteus aculeatus) and Eurasian perch (Perca fluviatilis) have been reported, however, the gut microbiota of the zebrafish is similar between sexes (Liu et al., 2016b). On the other hand, with the different studies, the underlying mechanisms are not yet understood.

There are still questions on whether genetics or the environment has greater influence on the gut microbiota. To date, host genetics has been considered the most significant factor in determining the fish gut microbiota.

Age/sexual maturity- Differences in the microbiota composition have been identified between juvenile and sexually mature individuals. Zebrafish juveniles gut have higher bacterial affluence than adult fish, suggesting an increase response of gut microbiota to higher levels of circulating sex hormone levels in adult compared to juvenile. In addition, the gut associated lymphoid tissue (GALT) may interact differently with the gut microbiota in 
juvenile and mature fish. Similarly, in southern catfish, gut microbial diversity increases with the host ages (Zhang et al., 2018).

The early developmental stages of fish showed changes in the gut microbiota. In Atlantic salmon (Salmo salar), intestinal microbiota compositions vary between embryonic stages. It is possible that modifications in diet added to the differences between juvenile and mature fish gut microbiota. Different ages of fish and sexual stages might have diverse nutrient requirements and adjust their diets and feeding rates to get sufficient energy. As the diet and gut microbiota composition change with age, the contribution of the gut microbiota to host homeostasis also changes. Though, further research is needed to explain this probable relationship between fish age, fish gut microbiota and energy homeostasis (Zhang et al., 2018).

Feeding habits/diet- Feeding habits also alter the structure and composition of the gut microbiota. Gut bacterial diversity is generally lower in carnivores, and increases in omnivores and herbivores. The most abundant bacteria in herbivorous fish include Clostridium, Citrobacter, and Leptotrichia, Cetobacterium and Halomonas (Li et al., 2014; Liu et al., 2016a; Kashinskaya et al., 2018). This has also been found in marine and freshwater fish, signifying that the trophic level is one of the most important factor affecting the gut microbiota composition.

The gut microbiota can also vary within species of the same trophic level. For example, the gut microbiota of four herbivorous Asian carp species (silver carp, Hypophthalmichthys molitrix; bighead carp, Hypophthalmichthys nobilis; grass carp, Ctenopharyngodon idella; and common carp, Cyprinus carpio) reared in the same environmental conditions exhibits inter-species differences (Li et al., 2018).
Severe dietary alterations, such as fasting also influence the fish gut microbiota. During times of fasting, morphological changes in the GIT occur due to the low nutrient uptake, which may influence the changes in the gut microbiota (Bruce et al., 2018). Besides, a depletion of nutrients induces change in gut microbiota composition to favor bacterial communities that use more varying energy sources and are able to survive under inadequate nutrient. In the Asian seabass, Lates calcarifer, fasting induces a significant enhancement of Bacteroidetes and a depletion of Betaproteobacteria (Xia et al., 2014).

The act of feeding itself can also control the microbiota. In rainbow trout, 3 hours after feeding is not sufficient to cause any considerable changes in the bacterial composition, but can cause changes in species richness and relative abundance (Mente et al., 2018). Similarly, in southern catfish, even if the diversity remains the same, the relative quantity of bacterial phyla differs at different hours of feeding, signifying that shortterm food digestion may alter community structure, but has less effect on the microbial composition (Zhang et al., 2018).

Fish age and growth: Age has a significant influence on congregation of microbiota in fish, particularly in the gut. Bacteria can colonize quickly in egg where different glycoproteins on the surface of egg may develop in speciesspecific microbiota. Bacterial diversity is found more during first feeding and its population is depending upon the nature of food items. The diversity is continuing with the age of the fish. Some common gut microbiota are also found may be due to surrounding aquatic environment (Zhang et. al., 2018).

Disease- Inadequate reports are available about the effect of disease on fish gut microbiota. Use of antimicrobial agent such as potassium permanganate $\left(\mathrm{KMnO}_{4}\right)$ is effective in 
disruption of the skin microbiota of channel catfish and increase susceptibility to columnaris disease caused by Flavobacterium columnare (Mohammed and Arias, 2015).

\section{Conclusion}

Several studies strongly propose that the fish gut microbiota influences the health of the fish with physiology, digestion, stress response, reproduction and the immune system. Comparatively a few studies on the effects of the microbiota on energy homeostasis have been conducted till date and large variations exist among the results. Studies on fish microbiome had provided comprehensive idea over proper understanding of host-microbe interactions like how microbes influence digestion, immune function, behavior and overall fish health. Although researchers working in this field have significantly expanded our knowledge on this topic there is still great scope for further research.

Diet and trophic level are the obvious influencing factors of fish gut microbial composition. Seasonal variation with the associated changes in water temperature and captive rearing has also been revealed to influence microbiota composition and some studies have cited the detrimental impact of each. In this context, strategies to enable the manipulation of gut microbiota composition for healthy microbiota are necessary. Probiotics and

\section{REFERENCES}

Al-Harbi AH and Uddin MN, 2004. Seasonal variation in the intestinal bacterial flora of hybrid tilapia (Oreochromis niloticus $\times$ Oreochromis aureus) cultured in earthen ponds in Saudi Arabia. Aquaculture, 229(1): 37-44, doi: 10.1016/ S0044-8486(03)00388-0

Avella MA, Place A, Du SJ, Williams E, Silvi S et al., 2012. Lactobacillus rhamnosus accelerates zebrafish backbone calcification and gonadal differentiation through effects on the GnRH and prebiotics are the forefront, though there is a lack of baseline compositional data from healthy wild fish in their natural environments. Therefore, an increased focus toward collecting such data is vital. The ultimate goal is to better understand the innate relationship between gut microbiota and their fish hosts.

Research in this field has considerably improved our knowledge on this topic though there is still great scope for further work. Data collection from wild populations, laboratory experiments and work within aquaculture will all be important contributors. Unquestionably next generation sequencing technologies will help this very much and provide more broad datasets. Effects of particular microbes and their associated components on fish host will develop the ability to manoeuvre and strengthen fish gut microbiota to enhance health and aquaculture productivity.

Conflict of interest: There is no conflict of interest regarding the present work.

\section{ACKNOWLEDGEMENTS}

The authors gratefully acknowledge the assistance extended by the Faculty of Fishery Sciences, West Bengal University of Animal and Fishery Sciences, West Bengal, India. Special thanks to National Bureau of Fish Genetic Resources (NBFGR), Lucknow, Uttar Pradesh, India and National Fisheries Development Board (NFDB), Hyderabad, Telangana, India.

IGF systems. PLoS One, 7: e45572, doi: 10.1371/ journal.pone.0045572

Bernier NJ, 2010. Food intake regulation and disorders. Fish Dis Disord, 2: 238-266, doi: $10.1079 / 9781845935535.0238$

Bolnick DI, Snowberg LK, Hirsch PE, Lauber CL, Org E et al., 2014. Individual diet has sex-dependent effects on vertebrate gut microbiota. Nat Commun, 5: 4500, doi: 10.1038/ncomms5500 
Bruce TJ, Neiger RD and Brown ML, 2018. Gut histology, immunology and the intestinal microbiota of rainbow trout, Oncorhynchus mykiss (Walbaum), fed process variants of soybean meal. Aquac Res, 49(1): 492-504, doi: 10.1111/are. 13480

Butt RL and Volkoff H, 2019. Gut microbiota and energy homeostasis in fish. Front Endocrinol, 10: 9, doi: 10.3389/fendo.2019.00009

Cantas L, Sorby JR, Alestrom P and Sorum H, 2012. Culturable gut microbiota diversity in zebrafish. Zebrafish, 9(1): 26-37, doi: 10.1089/ zeb.2011.0712

Carnevali O, Maradonna F and Gioacchini G, 2017. Integrated control of fish metabolism, wellbeing and reproduction: the role of probiotic. Aquaculture, 472: 144-155, doi: 10.1016/j.aquaculture.2016.03.037

Cheesman SE, Neal JT, Mittge E, Seredick BM and Guillemin K, 2011. Epithelial cell proliferation in the developing zebrafish intestine is regulated by the Wnt pathway and microbial signaling via Myd88. Proc Natl Acad Sci USA, 108(Suppl 1): 4570-4577, doi: 10.1073/pnas. 1000072107

Davis DJ, Bryda EC, Gillespie CH and Ericsson AC, 2016. Microbial modulation of behavior and stress responses in zebrafish larvae. Behav Brain Res, 311: 219-227, doi: 10.1016/ j.bbr.2016.05.040

Dehler CE, Secombes CJ and Martin SAM, 2017. Environmental and physiological factors shape the gut microbiota of Atlantic salmon parr (Salmo salar L.). Aquaculture, 467: 149-157, doi: 10.1016/j.aquaculture. 2016.07.017

Egerton S, Culloty S, Whooley J, Stanton C and Ross RP, 2018. The gut microbiota of marine fish. Front Microbiol, 9: 873, doi: 10.3389/ fmicb.2018.00873

Falcinelli S, Rodiles A, Hatef A, Picchietti S, Cossignani $\mathrm{L}$ et al., 2018. Influence of probiotics administration on gut microbiota core: A review on the effects on appetite control, glucose, and lipid metabolism. J Clin Gastroenterol, 52(Suppl 1): S50-56, doi: 10.1097/MCG. 0000000000001064
Fetissov SO, 2017. Role of the gut microbiota in host appetite control: bacterial growth to animal feeding behaviour. Nat Rev Endocrinol, 13:1125, doi: $10.1038 /$ nrendo.2016.150

Ghanbari M, Kneifel W and Domig KJ, 2015. A new view of the fish gut microbiome: advances from next-generation sequencing. Aquaculture, 448: 464-475, doi: 10.1016/j.aquaculture. 2015.06.033

Hoseinifar SH, Soleimani N and Ring $\varnothing$ E, 2014. Effects of dietary fructo-oligosaccharide supplementation on the growth performance, haemato-immunological parameters, gut microbiota and stress resistance of common carp (Cyprinus carpio) fry. Br J Nutr, 112(8): 12961302, doi: 10.1017/S0007114514002037

Kan H, Zhao F, Zhang X-X, Ren H and Gao S, 2015. Correlations of gut microbial community shift with hepatic damage and growth inhibition of Carassius auratus induced by pentachlorophenol exposure. Environ Sci Technol, 49(19): 11894-11902, doi: 10.1021/ acs.est.5b02990

Kashinskaya EN, Simonov EP, Kabilov MR, Izvekova GI, Andree KB et al., 2018. Diet and other environmental factors shape the bacterial communities of fish gut in an eutrophic lake. J Appl Microbiol, 125(6): 1626-1641, doi: 10.1111/jam. 14064

Kim S, Covington A and Pamer EG, 2017. The intestinal microbiota: antibiotics, colonization resistance, and enteric pathogens. Immunol Rev, 279(1): 90-105, doi: 10.1111/imr.12563

Lawley TD and Walker AW, 2013. Intestinal colonization resistance. Immunology, 138(1): 1-11, doi: 10.1111/j.1365-2567.2012.03616.x

Lazado CC and Caipang CMA, 2014. Mucosal immunity and probiotics in fish. Fish Shellfish Immunol, 39:78-89, doi: 10.1016/j.fsi.2014. 04.015

Li J, Ni J, Li J, Wang C, Li X et al., 2014. Comparative study on gastrointestinal microbiota of eight fish species with different feeding habits. J Appl Microbiol, 117(6): 1750-1760, doi: 10.1111/ jam. 12663 
Li X, Yu Y, Li C and Yan Q, 2018. Comparative study on the gut microbiotas of four economically important Asian carp species. Sci China Life Sci, 61: 696-705, doi: 10.1007/s11427-0169296-5

Li X, Zhou L, Yu Y, Ni J, Xu W et al., 2017. Composition of gut microbiota in the gibel carp (Carassius auratus gibelio) varies with host development. Microb Ecol, 74: 239-249, doi: 10.1007/ s00248-016-0924-4

Liu H, Guo X, Gooneratne R, Lai R, Zeng C et al., 2016a. The gut microbiome and degradation enzyme activity of wild freshwater fishes influenced by their trophic levels. Sci Rep, 6: 24340, doi: $10.1038 /$ srep24340

Liu Y, Yao Y, Li H, Qiao F, Wu J et al., 2016b. Influence of endogenous and exogenous estrogenic endocrine on intestinal microbiota in zebrafish. PLoS One, 11(10): e0163895, doi: 10.1371/journal.pone .0163895

Mehdinejad N, Imanpour MR and Jafari V., 2018. Combined or individual effects of dietary probiotic, Pediococcus acidilactici and nucleotide on reproductive performance in goldfish (Carassius auratus). Probiotics Antimicrob Proteins, 11(1): 233-238, doi: $10.1007 / \mathrm{s} 12602-017-9377-4$

Meng XL, Li S, Qin CB, Zhu ZX, Hu WP et al., 2018 Intestinal microbiota and lipid metabolism responses in the common carp (Cyprinus carpio L.) following copper exposure. Ecotoxicol Environ Saf, 160: 257-264, doi: 10.1016/j.ecoenv. 2018.05.050

Mente E, Nikouli E, Antonopoulou E, Martin SAM and Kormas KA, 2018. Core vs. diet -associated and postprandial bacterial communities of the rainbow trout (Oncorhynchus mykiss) midgut and feaces. Biol Open, 7(6): bio034397, doi: 10.1242/bio.034397

Mohammed $\mathrm{HH}$ and Arias CR, 2015. Potassium permanganate elicits a shift of the external fish microbiome and increases host susceptibility to columnaris disease. Vet Res, 46:82, doi:10.1186/ s13567-015-0215-y

Phelps D, Brinkman NE, Keely SP, Anneken EM, Catron TR et al., 2017. Microbial colonization is required for normal neurobehavioral development in zebrafish. Sci Rep, 7: 11244, doi: 10.1038/s41598-017-10517-5

Ribet D and Cossart P, 2015. How bacterial pathogens colonize their hosts and invade deeper tissues. Microbes Infect, 17(3): 173-183, doi: 10.1016/j.micinf.2015.01.004

Sandrini S, Aldriwesh M, Alruways $M$ and FreestoneP,2015. Microbial endocrinology: host-bacteria communication within the gut microbiome. J Endocrinol, 225(2): R21-34, doi: 10.1530/JOE-14-0615

Sekirov I and Finlay BB, 2009. The role of the intestinal microbiota in enteric infection. J Physiol, 587(17): 4159-4167, doi: 10.1113/ jphysiol.2009.172742

Semova I, Carten JD, Stombaugh J, Mackey LC, Knight $\mathrm{R}$ et al., 2012. Microbiota regulate intestinal absorption and metabolism of fatty acids in the zebrafish. Cell Host Microbe, 12(3): 277-288, doi: 10.1016/j.chom.2012.08.003

Sheng Y, Ren H, Limbu SM, Sun Y, Qiao Fet al., 2018. The presence or absence of intestinal microbiota affects lipid deposition and related genes expression in zebrafish (Danio rerio). Front Microbiol, 9: 1124, doi: 10.3389/ fmicb.2018.01124

Stephens WZ, Burns AR, Stagaman K, Wong S, Rawls JF et al., 2016. The composition of the zebrafish intestinal microbial community varies across development. ISME J, 10(3): 644-654, doi: 10.1038/ismej.2015.140

Sudo N, 2014. Microbiome, HPA axis and production of endocrine hormones in the gut. In: Microbial Endocrinology: The Microbiota-Gut-Brain Axis in Health and Disease. New York, NY: Springer pp177-194

Tapia-Paniagua ST, Reyes-Becerril M, Ascencio-Valle F, Esteban MÁ, Clavijo E et al., 2011. Modulation of the intestinal microbiota and immune system of farmed Sparus aurata by the administration of the yeast Debaryomyces hansenii L2 in conjunction with inulin. J Aquac Res Develop, S1: 012, doi: 10.4172/ 2155-9546.S1-012 
Tarnecki AM, Burgos FA, Ray CL and Arias CR, 2017. Fish intestinal microbiome: diversity and symbiosis unraveled by metagenomics. J Appl Microbiol, 123(1): 2-17, doi: 10.1111/ jam. 13415

Vatsos IN, 2016. Standardizing the microbiota of fish used in research. Lab Anim, 51(4): 353-364, doi: $10.1177 / 0023677216678825$

Wang AR, Ran C, Ringø E and Zhou ZG, 2018. Progress in fish gastrointestinal microbiota research. Rev Aquacult, 10: 626-640, doi: $10.1111 /$ raq. 12191

Wang W, Wu S, Zheng Y, Cheng Y, Li W et al., 2015. Characterization of the bacterial community associated with early-developmental stages of grass carp (Ctenopharyngodon idella). Aquac Res, 46(11): 2728-2735, doi: 10.1111/are.12428

Xia JH, Lin G, Fu GH, Wan ZY, Lee Met al., 2014. The intestinal microbiome of fish under starvation. BMC Genom, 15: 266, doi: 10.1186/1471-2164-15-266

Xiong Jin-Bo, Nie L and Chen J, 2019. Current understanding on the roles of gut microbiota in fish disease and immunity. Zool Res, 40(2): 7076, doi: 10.24272/j.issn.2095-8137.2018.069

Yan Q, Li J, Yu Y, Wang J, He Z et al., 2016. Environmental filtering decreases with fish development for the assembly of gut microbiota. Environ Microbiol, 18(12): 47394754, doi: 10.1111/1462-2920.13365

Zhang Z, Li D, Refaey MM, Xu W, Tang R et al., 2018. Host age affects the development of southern catfish gut bacterial community divergent from that in the food and rearing water. Front Microbiol, 9: 495, doi: 10.3389/fmicb. 2018.00495

Zhou L, Limbu SM, Qiao F, Du Z-Y and Zhang M, 2018. Influence of long-term feeding antibiotics on the gut health of Zebrafish. Zebrafish, 15(4): 340-348, doi: 10.1089/zeb.2017.1526

Zhou Z, Liu Y, Shi P, He S, Yao B et al., 2009. Molecular characterization of the autochthonous microbiota in the gastrointestinal tract of adult yellow grouper (Epinephelus awoara) cultured in cages. Aquaculture, 286(3): 184-189, doi: 10.1016/j.aquaculture.2008. 10.002

Received-30.08.2020, Accepted - 21.10.2020, Published - 01.12.2020 